\title{
PROGNOSTIC SIGNIFICANCE OF EPIDERMAL GROWTH FACTOR RECEPTOR (EGFR) IN HEAD AND NECK TUMOURS AT SOME SELECTED HOSPITAL IN GHANA
}

Precious Barnes ( $\nabla$ precious.barnes@ucc.edu.gh )

University of Cape Coast

F.A Yeboah

Kwame Nkrumah University of Science and Technology

Jinling Zhu

Jiamusi University School of Medicine

Roland Osei Saahene

Jiamusi University School of Medicine

Patrick Akakpo

University of Cape Coast

Richard K.D Ephrraim

University of Cape Coast

Research article

Keywords: EGFR, Head and neck tumour, Clinicopathological factors

Posted Date: July 31st, 2019

DOI: https://doi.org/10.21203/rs.2.12130/v1

License: (c) (i) This work is licensed under a Creative Commons Attribution 4.0 International License.

Read Full License 


\section{Abstract}

Abstract: Background: Head and neck Tumour (HNT) are tumour of the paranasal sinuses, the salivary glands and the upper aero-digestive tract. EGF can stimulate cell proliferation, cell differentiation, cell growth, migration, and inhibit apoptosis. The aim of this study was to investigate the expression of EGFR in head and neck tumour. Method: EGFR expression was analyzed using quantitative real-time PCR (qRTPCR) and immunohistochemical staining on tissue samples from a consecutive series of 150 head and neck tumour patients who underwent tumor resections between 2014 and 2018. Results: The relationship between EGFR expressions, clinicopathological factors, was investigated. qRT-PCR results showed that the EGFR expression was high in tumor tissue samples than in the normal head and neck tissues. High and low EGFR was compared with ages $\leq 40,>40$ in the head and neck cancer of $p$-value 0.03 . There was a significant difference between the histological differentiation of the malignant tumour with $p$ values of 0.001 , when well, moderate and poor was compared. There was a significant difference ( $p$-value 0.012) between the I-II and III-IV tumour stages when the high level and low expression of EGFR were compared. Conclusion: Our data suggest that EGFR plays an important role in head and neck tumour progression and that its expression will provide baseline data to facilitate identification of new molecular targeting therapeutics. Keywords: EGFR, Head and neck tumour, Clinicopathological factors.

\section{Background}

Head and neck tumours (HNT) are tumours that originate from the oral cavity, pharynx and larynx. It is one of the most common tumours with 599,000 patients diagnosed worldwide in 2012 and 325,000 deaths [1].

Epidermal growth factor receptor (EGFR) is classified as part of the ErbB/HER family of receptor tyrosine kinase (TK). The EGFR is a transmembrane glycoprotein. These receptors comprise of an extracellular ligand-binding domain, a hydrophobic transmembrane segment, and an intracellular TK domain. A change in the conformation of EGFR occurs when it is bound to ligands such as amphiregulin and transforming growth factor alpha (TGF-a). Homo- or hetero-dimerization with other ErbB/HER family of receptors and results to autophosphorylation and its activation [2]. The activation of EGFR initiates the induction of intracellular signaling pathways which initiate cell proliferation, invasion, angiogenesis and metastasis during tumourgenesis.

Elevated EGFR are seen occurs in many epithelial malignancies such as head and neck tumours. This study investigated the correlation of EGFR expression with clinicopathological parameters such as age, sex, tumour differentiation and the stage of the tumour for development of head and neck tumour.

\section{Methods}

Patient Tissue Samples 
A total of 150 paraffin-embedded blocks of samples (archival tissue specimens) from patients with histologically HNT specimens were collected from the pathology laboratory at Cape Coast Teaching Hospital and KATH in Ghana. 150 normal tissues were also taken as a control. The clinical parameters are indicated in Table 1and 2. This study was approved by the Institutional Ethics Committee of the Cape Coast Teaching Hospital and theCommittee of Human Research, Publications and Ethics at kwame Nkrumah University of Science and Technology, School of Medical Sciences \& Komfo Anokye Teaching Hospital.

\section{Immunohistochemistry}

Epidermal Growth factor receptor (EGFR) expressions were detected by immunohistochemistry (IHC). The goat anti-EGFR (Biosynthesis Biotechnology Co LTD, Beijing China) antibody was used. 4um formalin fixed paraffin embedded (FFPE) mounted sections were deparaffinized, rehydrated and rinsed three times in distilled water. After heat antigen retrieval and cooling in citrate buffer solution, they were incubated with $5 \%$ bovine serum albumin (BSA) at $37^{\circ} \mathrm{C}$ for 30 minutes following three times phosphate-buffered saline (PBS) rinses. The slides were incubated at $4^{\circ} \mathrm{C}$ with primary antibody EGFR (1:500 dilution) overnight followed by incubation with Biotinylated anti-rabbit immunoglobulin $\mathrm{G}(\mathrm{IgG})$ and $\mathrm{SABC}$ at $37^{\circ} \mathrm{C}$ for 30 minutes each. The subsequent processes were performed as previously described [3].

The EGFR in the cells were scored semi-quantitatively according to the number of positive-staining cells and the staining intensity. Cytoplasmic immunostaining in the tumor cells was considered positive staining.

\section{Real-time PCR}

RNA was obtained from the tumor with a TRIzol reagent (Takara, Otsu, Japan). $0.5 \mathrm{mg}$ total RNA from each sample were used for the Reverse transcription. SYBR Green PCR Master Mix (Takara) polymerase chain reaction. The sequences of the primer pairs of EGFR were as follows: Forward 5'GCGTCTCTTGCCGGAATGT-3' Reverse 5'-GGCTCACCCTCCAGAAGGTT-3'. The levels of EGFR were presented as $\triangle \mathrm{Ct}=\mathrm{Ct}$ of EGFR-Ct of GAPDH, and 2- $\triangle \triangle \mathrm{Ct}$ method.

\section{Statistical analysis}

SPSS 17.0 for Windows (SPSS, Chicago, IL) is the statistical tool which was used. The statistical analyses were performed using. Significant level was set at 0.05 . For qualitative variables, proportions and percentages was used to summarize the data. The relationship between EGFR expression and HNT patient clinicopathologic parameters were analysed with $\chi 2$ test.

\section{Results}


In our study, during the period of 4 years, from 2014 to 2016, a total of 250 cases of head and neck tumour were studied. Epidermal growth factor receptor mutation analysis was done for 150 head and neck normal (control) tissues and 150 head and neck tumours. Age of the patients varied from 18 to 89 years.

\section{Clinical and pathologic variable analysis for Head and Neck Malignant Tumour}

Out of the 150 EGFR analysis, 98 of the cases were patients with head and neck malignancy and 52 were patient with benign head and neck tumour. $p$ value for the EGFR scoring when high and low EGFR was compared was 0.03 (Table 1), which was significant statistically. Out of 98 malignant tumour of head and neck cancer cases in the study, 62 and 36 were males and females respectively (Table 1). For both males and females, majority of the cases had a high EGFR 40(58.8\%) males and 28(\%) females). But no association was found between sex and EGFR scoring (Table 1).

When the high and low levels of EGFR were analysed, oral cavity (36.7\%) was the most prevalent tumour location in our study, followed by respiratory (28.6\%), mandible (14.2\%), nasopharynx (11.2\%) (Table 1). All the anatomical sites were presented with a majority of high expression levels of EGFR.

\section{The level of EGFR and Tumour Staging}

When the retrievable staging information of the patients was analysed, the majority were in stage II $37(37.8 \%$ ) tumour stage, followed by stage III 25(25.5\%). From this present study, III and III-IV, EGFR high expression were found in $41(60.3 \%)$ and $27(39.7 \%)$ cases, respectively (Table 1). There was significant difference ( $p$-value 0.012 , Table 1$)$ when the stages I-II and III-IV were compared.

The high levels of EGFR measured by the overall score was presented in $32.4 \%, 29.4 \%, 7.4 \%, 16.2 \%, 11.8$, $1.47 \%$ and $1.47 \%$ of cases from oral cavity, respiratory, upper $\mathrm{GI}$, mandible, nasopharynx, salivary gland and the eye, respectively (Table 1), with an overall rate of high-expression at $45.3 \%$ (Table 3 ).

Based on the histological grade, when the high and low levels of EGFR were compared most (46.9\%) of the patients were diagnosed as moderate differentiated HNC followed by $36.7 \%$ well differentiated and $16.3 \%$ poorly differentiated. When well and moderate were compared with poor differentiation there was a significant difference of p-value 0.001. Twenty-five (25.5\%) were stage I, 22 (22.4\%) stage II, 14 (14.3\%) stage III and $37(37.8 \%)$ stage IV. There was a significant difference between the high and low expression of the EGFR ( $p$-value 0.012) when I-II and III-IV were compared. 


\section{Clinical and pathologic variable analysis for Head and Neck Benign Tumour}

There were 52 patients with head and neck benign tumour. 34(65.4) were above 40 years old and 18 (34.6\%) below 40 years old. When the high and low expression of EGFR was compared with the ages between above and below 40 years, there was a significant difference of p-value 0.013 . Twenty- eight (53.8\%) were male, 24 (46.1) were female (Table 2).

\section{Immunohistochemistry Epidermal Growth Factor Receptor Protein Expression}

The protein levels of the epidermal growth factor receptor were analysed by immunohistochemistry. The EGFR were seen in the cytoplasmic and also in the nucleus (Figure 2). Tumours with EGFR expression $<20.8$ were reported as low $(n=49)$, and tumours with EGFR expression $>20.8$ were reported as high $(n=$ 101).

\section{Expression of EGFR of the Benign Tumour}

The males were 28 and the females were 24 . The ages were between 18-89 years. Among the benign tumours, a high expression of EGFR was found in 10 of 19 (\%) cases from oral cavity, 14 of 18 (\%) cases from mandible, 7 of 12 (\%) cases from respiratory, 2 of 3 nasopharynx (Table 2). The overall high expression of EGFR in benign tumour is 33 (63.5\%) (Table 3$)$.

\section{The levels of EGFR and Clinical Parameters of the Benign Tumours}

The level of EGFR correlated with ages (aged $\leq 40$ years vs. $>40$ years) p-value 0.013 (Table 2). But no association was found with sex (28 males and 24 female) Table 2 ( $p$-value 0.89).

\section{Discussion}

Head and neck region having geographical variation in the incidence and anatomic distribution of HNT worldwide. Mitogenic EGFR signaling pathways and its ligand, transforming growth factor-alpha (TGFalpha) in HNT are usually highly expressed. It is relatively less studied particularly in Ghanaian population within the African populations. Moreover, there is no local study in Ghana about the level of EGFR expression in head and neck tumours in Ghana. This study investigated the level of expression of EGFR in head and neck tumours in some selected hospital in Ghana. Regards with this study, a high 
expression of EGFR staining was found in 101(67.3\%) head and neck tumours (Table 3). This is in accordance with a study, which reported high expression of EGFR in $42-80 \%$ of the cases [5]. From this present study, high expression of EGFR was seen among both malignant and benign tumour of patients with head and neck (Table 3). But the level of EGFR expression is negligible in normal head and neck tissue (Table 3, Figure 2B).

The abnormal expression of EGFR is as a results of high expression of EGFR in normal epithelium which is adjacent to tumour and abnormal up-regulation in the change from dysplasia to squamous cell carcinoma [6]. This results from an uncontrolled ligand production, receptor mutations or deletions which results in abnormal cell multiplication, invasion, high angiogenesis and metastasis. Activating the EGFR leads to induction of cellular pathway which is initiated by toxic environmental stimuli such as ultraviolet irradiation, or to receptor occupation by EGF, the EGFR forms homo- or heterodimers with other family members [7].

The high expression of EGFR in head and neck tumours also leads to tumour invasion since it initiates the produces proteolytic enzyme-matrix metalloproteinases through paracrine and autocrine epidermal growth factor (EGF) or transforming growth factor-alpha (TGF-a)-EGFR system [8].

From this present study the EGFR was found localized in both the nucleus and the cytoplasm (Figure 2) this is similar to a study by Lo et al [8]. In the normal tissue, EGFR is localized at the cytoplasm (Figure 2B), this explain the fact that EGFR signaling is involve in cell proliferation.

Nuclear localization sequences and endocytosis cause the nuclear translocation of the EGFR. EGFR carried to nucleus instead of degraded. When there is a cytoplasmic-nuclear transport, EGFR proteins which have nuclear localization sequences (NLS) form a complexes with importin $\alpha / \beta$. The complex then associates with nucleoporins in the nuclear pore complex (NPC) and finally the EGFR is transported into the nucleus [9].

In the nucleus, it serves as a transcriptional regulator which transmits signals and inititaes cell proliferation, tumor progression, DNA repair and replication, chemo- and radio-resistance. Nuclear EGFRis a a transcriptional co-activator for cyclin D1[10], nitric oxide synthase (iNOS) [11], B-Myb [12, 13], cyclooxygenase-2 (COX-2) [14], c-Myc [15] and breast cancer resistant protein (BCRP) [16].

From this study, high levels of EGFR significantly associated with the stages of the head and neck tumours when stage I-II and III-IV (p-value 0.012) (Table 1), this was in accordance with the study by Reimers et al. [17] which also suggested a correlation of EGFR expression with advanced tumor stage. This also confirm the role of EGFR expression in relation to tumour growth and size [18]. Moreover, report also indicates that lymph node and advanced stage IV disease is a prognostic parameter for overall survival [19].

\section{Conclusion}


Epidermal Growth Factor Receptor can serve as biomaker in diagnosing and prognosis of head and neck tumour. Hence, EGFR can be use in developing targeting therapy for head and neck tumour.

\section{List Of Abbreviation}

HNT- Head and Neck Tumour

EGFR-Epidermal Growth Factor Receptor

TK- Tyrosine Kinase

TGF-a- transforming growth factor alpha

HNSCC-Head and Neck Squamous Carcinoma

KATH-Komfo Anokye Teaching Hospital

FFPE -Formalin fixed paraffin embedded

PBS - Phosphate-buffered saline

\section{Declarations}

\section{Ethical approval and consent to participate}

The Institutional Review Board of the Kwame Nkrumah University of Science and Technology gave their approval for the study (Ethical Clearance ID: CHRPE/AP/609/18) and also the Ethical Review Board of Cape Coast Teaching Hospital gave approval for the commencement of the research (Ethical Clearance ID: CCTHERC/RS/EC/2017/52).

\section{Consent for publication}

\section{N/A}

\section{Availability of data and materials}

The datasets used will be deposited in the archives of Kwame Nkrumah University of Science and Technology Library and is available from the corresponding author on reasonable request. 


\section{Competing interests}

The authors confirmed that there is no competing interest.

\section{Funding}

The study was funded by University of Cape Coast

\section{Authors' contributions}

PB, PA and ROS-conceived the study, contributed to the study design, data collection and analysis, manuscript preparation. FAY, RED- contributed to the preparation and finalization of the manuscript and data analysis and also contributed in the laboratory analysis. JZ performed the RT-PCR to determine the EGFR expression.

\section{Acknowledgements}

We would like to acknowledge the help of all the Pathologist of Cape Coast Teaching Hospital.

\section{References}

1.Ferlay J, Shin HR, Bray F, Forman D, Mathers C, Parkin DM: Estimates of worldwide burden of cancer in 2008: GLOBOCAN 2008. Int J Cancer 2010, 127(12):2893-2917.

2.Ciardiello F, Tortora G: Epidermal growth factor receptor (EGFR) as a target in cancer therapy: understanding the role of receptor expression and other molecular determinants that could influence the response to anti-EGFR drugs. Eur J Cancer 2003, 39(10):1348-1354.

3.Saahene R, Wang J, Wang M-L, Agbo E, Song H: The role of CXC chemokine ligand 4/CXC chemokine receptor 3-B in breast cancer progression. Biotech Histochem 2018:1-7.

4.Van Oijen M, Rijksen G, Broek Ft, Slootweg P: Increased expression of epidermal growth factor receptor in normal epithelium adjacent to head and neck carcinomas independent of tobacco and alcohol abuse. Oral Dis 1998, 4(1):4-8.

5.Wen Q, Nishimura T, Miwa T, Nagayama I, Furukawa M: Expression of EGF, EGFR and PCNA in laryngeal lesions. The Journal of Laryngology \& Otology 1995, 109(7):630-636.

6.Shin DM, Ro JY, Hong WK, Hittelman WN: Dysregulation of epidermal growth factor receptor expression in premalignant lesions during head and neck tumorigenesis. Cancer Res 1994, 54(12):3153-3159. 
7.Rikimaru K, Tadokoro K, Yamamoto T, Enomoto S, Tsuchida N: Gene amplification and overexpression of epidermal growth factor receptor in squamous cell carcinoma of the head and neck. Head Neck 1992, 14(1):8-13.

8.Kusukawa J, Harada H, Shima I, Sasaguri Y, Kameyama T, Morimatsu M: The significance of epidermal growth factor receptor and matrix metalloproteinase -3 in squamous cell carcinoma of the oral cavity. European Journal of Cancer Part B: Oral Oncology 1996, 32(4):217-221.

9. Harel A, Forbes DJ: Importin beta: conducting a much larger cellular symphony. Mol Cel/ 2004, 16(3):319-330.

10.Lin S-Y, Makino K, Xia W, Matin A, Wen Y, Kwong KY, Bourguignon L, Hung M-C: Nuclear localization of EGF receptor and its potential new role as a transcription factor. Nat Cell Biol 2001, 3(9):802.

11.Lo H-W, Hsu S-C, Ali-Seyed M, Gunduz M, Xia W, Wei Y, Bartholomeusz G, Shih J-Y, Hung M-C: Nuclear interaction of EGFR and STAT3 in the activation of the iNOS/NO pathway. Cancer Cell 2005, 7(6):575589.

12.Hanada N, Lo HW, Day CP, Pan Y, Nakajima Y, Hung MC: Co-regulation of B-Myb expression by E2F1 and EGF receptor. Molecular Carcinogenesis: Published in cooperation with the University of Texas MD Anderson Cancer Center 2006, 45(1):10-17.

13.Hung L-Y, Tseng JT, Lee Y-C, Xia W, Wang Y-N, Wu M-L, Chuang Y-H, Lai C-H, Chang W-C: Nuclear epidermal growth factor receptor (EGFR) interacts with signal transducer and activator of transcription 5 (STAT5) in activating Aurora-A gene expression. Nucleic Acids Res 2008, 36(13):4337-4351.

14.Lo H-W, Cao X, Zhu H, Ali-Osman F: Cyclooxygenase-2 is a novel transcriptional target of the nuclear EGFR-STAT3 and EGFRvIII-STAT3 signaling axes. Mol Cancer Res 2010:1541-7786. MCR-1509-0391.

15.Jaganathan S, Yue P, Paladino DC, Bogdanovic J, Huo Q, Turkson J: A functional nuclear epidermal growth factor receptor, SRC and Stat3 heteromeric complex in pancreatic cancer cells. PLoS One 2011, 6(5):e19605.

16. Huang W-C, Chen Y-J, Li L-Y, Wei Y-L, Hsu S-C, Tsai S-L, Chiu P-C, Huang W-P, Wang Y-N, Chen C-H: Nuclear translocation of epidermal growth factor receptor by Akt-dependent phosphorylation enhances breast cancer-resistant protein expression in gefitinib-resistant cells. J Biol Chem 2011, 286(23):2055820568.

17.Reimers N, Kasper HU, Weissenborn SJ, Stützer H, Preuss SF, Hoffmann TK, Speel EJM, Dienes HP, Pfister HJ, Guntinas-Lichius 0: Combined analysis of HPV-DNA, p16 and EGFR expression to predict prognosis in oropharyngeal cancer. Int J Cancer 2007, 120(8):1731-1738.

18.Kawamoto T, Takahashi K, Nishi M, Kimura T, Matsumura T, Taniguchi S: Quantitative assay of epidermal growth factor receptor in human squamous cell carcinomas of the oral region by an avidin- 
biotin method. Jpn J Cancer Res 1991, 82(4):403-410.

19.Grandi C, Boracchi P, Mezzanotte G, Squadrelli M, Marubini E, Molinari R: Analysis of prognostic factors and proposal of a new classification for nasopharyngeal cancer. Head Neck 1990, 12(1):31-40.

\section{Tables}

Due to technical limitations the tables could not be inserted here. They can be found in the supplemental files.

\section{Figures}

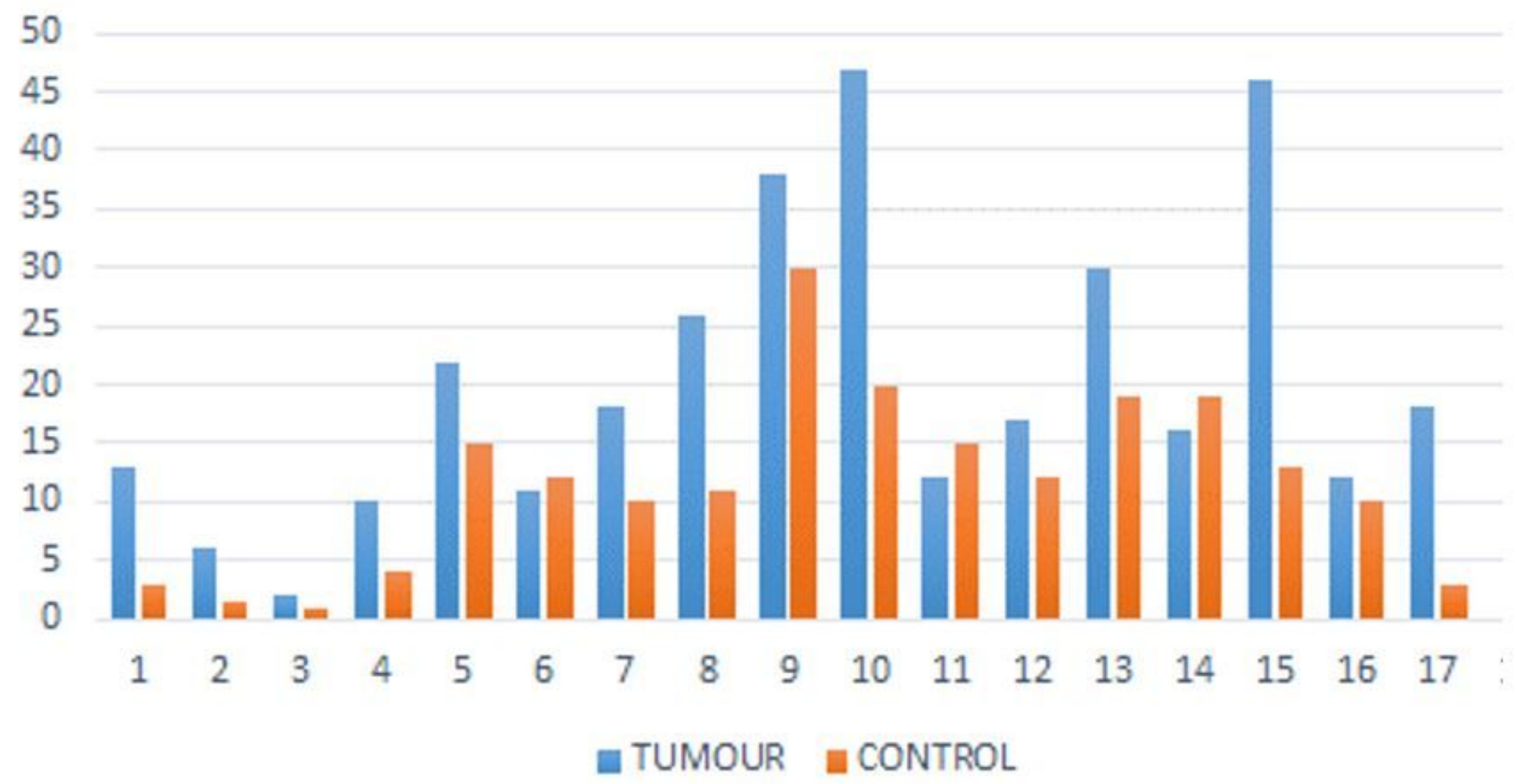

\section{Figure 1}

Real-time polymerase chain reaction analyses of EGFR in head and neck tumours samples and control tissues. The data are representation of mean \pm SD of three replicates in three different experiment. 

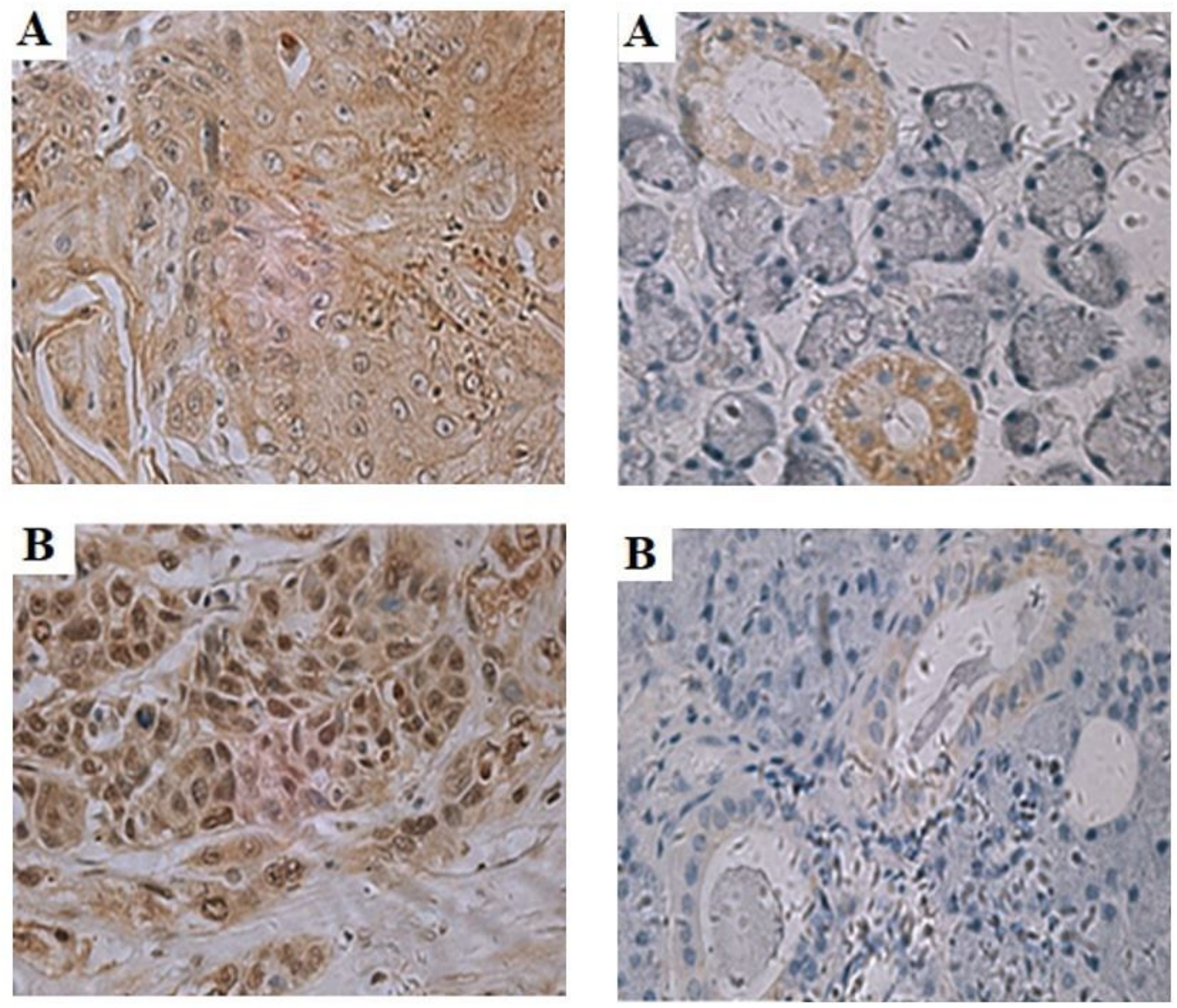

\section{Figure 2}

EGFR expression levels were upregulated in the head and neck tumour samples (left) compared with the paired control tissues (right). The data was produced from 3-5 fields per slide $\times 400$.

\section{Supplementary Files}

This is a list of supplementary files associated with this preprint. Click to download.

- TableforEGFR.docx 